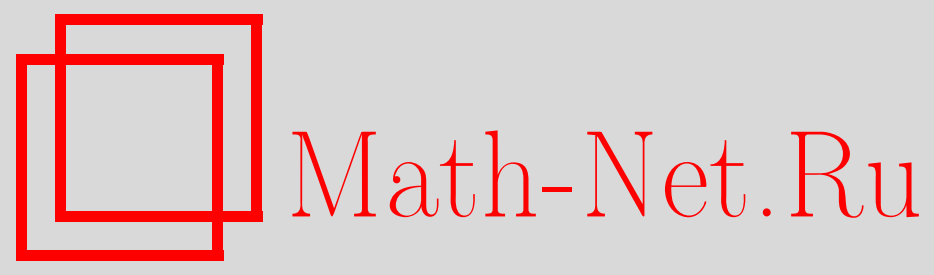

Н. Ф. Валеев, Регулярные решения многопараметрической обратной спектральной задачи, Матем. заметки, 2009, том 85, выпуск 6, 940-943

DOI: https://doi.org/10.4213/mzm7663

Использование Общероссийского математического портала Math-Net.Ru подразумевает, что вы прочитали и согласны с пользовательским соглашением http://www . mathnet.ru/rus/agreement

Параметры загрузки:

IP : 54.198 .187 .58

26 апреля 2023 г., 15:48:38

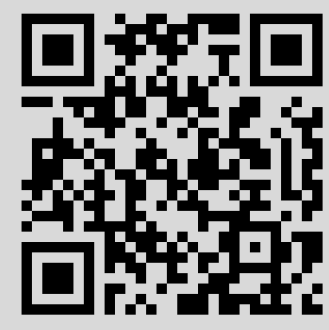




\section{Регулярные решения многопараметрической обратной спектральной задачи}

\section{Н. Ф. Валеев}

1. Многопараметрическая обратная спектральная задача. Пусть в $n$-мерном евклидовом пространстве $E^{n}$ задано семейство $m$-параметрических операторов вида

$$
B(\vec{p}, \lambda)=B_{0}(\lambda)+p_{1} B_{1}(\lambda)+p_{2} B_{2}(\lambda)+\cdots+p_{m-1} B_{m-1}(\lambda)+p_{m} B_{m}(\lambda),
$$

где $\vec{p}=\left(p_{1}, p_{2}, \ldots, p_{m}\right) \in \mathbb{C}^{m}$, линейные операторы $B_{k}(\lambda): E^{n} \rightarrow E^{n}$ аналитически зависят от спектрального параметра $\lambda \in \mathbb{C}$. При этих условиях число $\lambda \in \mathbb{C}$ будем называть собственным значением оператора $B(\vec{p}, \lambda)$, если оператор $[B(\vec{p}, \lambda)]^{-1}$ не существует.

ПОСТАНОВКА МНОГОПАРАМЕТРИЧЕСКОЙ ОБРАТНОЙ СПЕКТРАЛЬНОЙ ЗАДАЧИ (МПОСЗ). Требуется найти возможные значения вектора $\vec{p}$ из пространства $\mathbb{C}^{m}$, при которых наперед заданные числа $\lambda_{1}, \lambda_{2}, \ldots, \lambda_{m}$ являются собственными значениями оператора $B(\vec{p}, \lambda)$. При этом набор чисел $\lambda_{1}, \lambda_{2}, \ldots, \lambda_{m}$ будем называть спектральными данными и обозначать $\vec{\lambda}$.

Сформулированная постановка задачи часто возникает в математических моделях диагностики или идентификации технических систем по их собственным колебаниям. Аналогичные постановки обратных спектральных задач полезны также при исследовании моделей управления резонансными характеристиками объекта, когда посредством подбора доступных параметров объекта (линейной динамической системы) требуется придать этой системе те или иные частотно-резонансные характеристики. Этим задачам посвящено большое количество работ, см., например, работу [1] и библиографию к ней.

Поскольку МПОСЗ тесно связана с прикладными техническими проблемами, то ее решения соответственно должны быть устойчивыми к малым возмущениям (погрешностям измерений) матриц $B_{k}(\lambda)$ и спектральных данных $\vec{\lambda}$ задачи. В связи с этим введем важное понятие. Обозначим $b(\vec{p}, \lambda)=\operatorname{det}(B(\vec{p}, \lambda))$, тогда решение МПОСЗ эквивалентно решению системы из $k$ уравнений

$$
b\left(\vec{p}, \lambda_{k}\right)=0, \quad k=1, \ldots, m .
$$

Пусть $\overrightarrow{e_{j}}$ - ортонормированный базис пространства $E^{n}$ и

$$
W(\vec{p}, \vec{\lambda})=\sum_{j=1}^{n} b\left(\vec{p}, \lambda_{j}\right) \overrightarrow{e_{j}}
$$

Решение МПОС $\vec{p}^{*}$, соответствующее спектральным данным $\vec{\lambda}=\vec{\lambda}^{*}$, будем называть регулярным, если

$$
\operatorname{det} \frac{d W}{d \vec{p}}\left(\vec{p}^{*}, \vec{\lambda}^{*}\right) \neq 0 .
$$

Прямое исследование существования регулярных решений системы, их количества и, тем более, построение алгоритмов, гарантирующих нахождение всех ее решений для системы алгебраических уравнений (2), в общем случае затруднительны. Причиной тому является довольно сложный вид системы полиномиальных уравнений от переменных $p_{j}$.

Работа выполнена при поддержке Российского фонда фундаментальных исследований (грант № 08-01-97020). 
В связи с этим, следуя работам [2] и [3], преобразуем систему (2) к виду, в котором координаты вектора $\vec{p}=\left(p_{1}, p_{2}, \ldots, p_{m}\right)$ окажутся "разделенными" между собой. Говоря точнее, мы построим полиномы от одной переменной для каждой координаты вектора управления.

Обозначим через $H_{m}=E^{n} \otimes E^{n} \otimes \cdots \otimes E^{n}$ тензорное произведение $m$ экземпляров евклидовых пространств $E^{n}$. Пусть $\Omega$ - множество всех перестановок чисел $\{1,2, \ldots, m\}$. Если $\omega=\left\{i_{1}, i_{2}, \ldots, i_{m}\right\} \in \Omega$, положим $\omega(k)=i_{k}$, а через $I(\omega)$ обозначим количество беспорядков (инверсий) в перестановке $\omega$. Введем в рассмотрение оператор $\Delta_{0}: H_{m} \mapsto$ $H_{m}$ :

$$
\Delta_{0}=\sum_{\omega \in \Omega}(-1)^{I(\omega)} B_{\omega(1)}\left(\lambda_{1}\right) \otimes B_{\omega(2)}\left(\lambda_{2}\right) \otimes \cdots \otimes B_{\omega(m)}\left(\lambda_{m}\right) .
$$

Теперь, полагая $B_{k}\left(\lambda_{j}\right)=B_{0}\left(\lambda_{j}\right)$ в формуле для $\Delta_{0}$, определим операторы

$$
\Delta_{k}: H_{m} \mapsto H_{m}, \quad k=1, \ldots, m .
$$

Операторы $\Delta_{k}: H_{m} \mapsto H_{m}, k=0, \ldots, m$, подробно изучены в работах [2] и [3].

Теорема 1. Пусть $\operatorname{rank}\left(\Delta_{0}\right)=N_{1}$, тогда МПОСЗ для оператора (1) имеет ровно $N_{1}$ регулярных решений.

Введем в рассмотрение ортогональные проекторы $G_{k}$ на подпространства

$$
V_{k}=\operatorname{Ker}\left(\Delta_{k}\right) \cap \operatorname{Ker}\left(\Delta_{0}\right)
$$

и полиномы от одной переменной:

$$
f_{k}(s)=\operatorname{det}\left((-1)^{k} \Delta_{k}+s \Delta_{0}+G_{k}\right), \quad k=1, \ldots, m .
$$

Следующее утверждение позволяет конструктивно строить все регулярные решения МПОС3.

Теорема 2. Пусть вектор $\vec{p}=\left(p_{1}, \ldots, p_{m}\right)$ - регулярное решение МПОСЗ для опеpaтора (1), тогда $f_{k}\left(p_{k}\right)=0$.

Таким образом, построение регулярных решений МПОСЗ сводится к поиску нулей многочленов $f_{k}(s)$.

2. МПОСЗ для операторов Штурма-Лиувилля. Рассмотрим дискретный оператор Штурма-Лиувилля:

$$
-\frac{y_{i-1}-2 y_{i}+y_{i+1}}{h^{2}}+p_{i} q_{i} y_{i}=\lambda y_{i}
$$

где $0<i<m+1$, а граничные условия имеют вид $y_{0}=0, y_{m+1}=0$.

Для этого оператора можно рассматривать задачу о восстановлении потенциала этого оператора $q=\left(p_{1} q_{1}, p_{2} q_{2}, \ldots, p_{m} q_{m}\right)$ по $m$ собственным значениям $\lambda_{1}<\lambda_{2}<\cdots<\lambda_{m}$. Данная задача легко приводится к МПОСЗ для оператора вида (1) и при этом

$$
\operatorname{rank}\left(\Delta_{0}\right)=m !
$$

Это означает, что, во-первых, многопараметрическая обратная спектральная задача разрешима для оператора (3) с любым набором собственных значений $\lambda_{1}<\lambda_{2}<\cdots<\lambda_{m}$; во-вторых, МПОСЗ имеет ровно $m$ ! регулярных решений.

МПОСЗ для дифференциальных операторов в гильбертовых пространствах можно исследовать с помощью теорем предыдущего пункта, используя некоторые методы теории вращения компактных векторных полей (см. [4]).

Рассмотрим самосопряженный оператор Штурма-Лиувилля:

$$
-y^{\prime \prime}(x, \lambda)+\lambda^{2} q(x, \vec{p}) y(x, \lambda)=0
$$


с потенциалом вида

$$
q(x, \vec{p})=\sum_{k=1}^{m} p_{k} q_{k}(x), \quad q_{k}(x) \in C[0,1],
$$

и граничными условиями Дирихле $y(0)=y(\pi)=0$.

Как и выше, рассмотрим задачу восстановления по спектральным данным $\lambda_{1}<\lambda_{2}<$ $\cdots<\lambda_{m}$ потенциала $q(x, \vec{p})$. Для исследования существования изолированных решений $\vec{p}$ обратной спектральной задачи для оператора (4) можно воспользоваться проекционными методами решения операторных уравнений. Тогда исходная задача будет сведена к МПОСЗ в конечномерном пространстве. Отсюда вытекает следующее утверждение.

Теорема 3. Пусть при каждом $\vec{p} \in \mathbb{C}^{m},\|\vec{p}\|=1$, бункция $q(x, \vec{p})=\sum_{k=1}^{m} p_{k} q_{k}(x)$ на $[0,1]$ имеет конечное число нулей, тогда у МПОСЗ для оператора (4) существуют изолированные решения.

Аналогичные результаты справедливы и для самосопряженных дифференциальных операторов более высокого порядка. Также эту теорему можно переформулировать для самосопряженных дифференциальных операторов в частных производных.

Теперь рассмотрим задачу о восстановлении коэффициентов граничных условий оператора Штурма-Лиувилля. Пусть задан оператор Штурма-Лиувилля:

$$
-\left(a(x) y^{\prime}(x, \lambda)\right)^{\prime}+\left(\lambda^{2} b(x)+c(x)\right) y(x, \lambda)=0,
$$

с граничными условиями

$$
y^{\prime}(0, \lambda)+\left(p_{1}+\lambda^{2} p_{2}\right) y(0, \lambda)=0, \quad-y^{\prime}(1, \lambda)+\left(p_{3}+\lambda^{2} p_{4}\right) y(1, \lambda)=0 .
$$

На коэффициенты $a(x), b(x)$ и $c(x)$ наложим следующие условия:

$$
a(x) \in C^{1}[0,1], \quad a(x)>0, \quad b(x) \in C[0,1], \quad b(x)>0, \quad c(x) \in C[0,1], \quad c(x)>0 .
$$

Предположим, что нам известны четыре собственных значения $\lambda_{1}, \lambda_{2}, \lambda_{3}, \lambda_{4}$ данного оператора, и рассмотрим задачу восстановления по этим собственным значениям вектора управления $\vec{p}=\left(p_{1}, p_{2}, p_{3}, p_{4}\right)$. Обозначим через $u(x, \lambda), v(x, \lambda)$ два линейно-независимых решения уравнения (5), удовлетворяющих начальным условиям

$$
u(0, \lambda)=1, \quad v(0, \lambda)=0, \quad u^{\prime}(0, \lambda)=0, \quad v^{\prime}(0, \lambda)=1 .
$$

Введем в рассмотрение следующие функции от собственных значений оператора (5):

$$
\begin{gathered}
f_{1}\left(\lambda_{k}, \lambda_{j}, \lambda_{i}\right)=\operatorname{det}\left(\begin{array}{ccc}
\left(v^{\prime}\left(1, \lambda_{k}\right)\right. & v\left(1, \lambda_{k}\right) & \lambda_{k}^{2} v\left(1, \lambda_{k}\right) \\
v^{\prime}\left(1, \lambda_{j}\right) & v\left(1, \lambda_{j}\right) & \lambda_{j}^{2} v\left(1, \lambda_{j}\right) \\
v^{\prime}\left(1, \lambda_{i}\right) & v\left(1, \lambda_{i}\right) & \lambda_{i}^{2} v\left(1, \lambda_{i}\right)
\end{array}\right), \\
f_{2}\left(\lambda_{k}, \lambda_{j}, \lambda_{i}\right)=\operatorname{det}\left(\begin{array}{ccc}
\left(u\left(1, \lambda_{k}\right)\right. & v\left(1, \lambda_{k}\right) & \lambda_{k}^{2} v\left(1, \lambda_{k}\right) \\
u\left(1, \lambda_{j}\right) & v\left(1, \lambda_{j}\right) & \lambda_{j}^{2} v\left(1, \lambda_{j}\right) \\
u\left(1, \lambda_{i}\right) & v\left(1, \lambda_{i}\right) & \lambda_{i}^{2} v\left(1, \lambda_{i}\right)
\end{array}\right) .
\end{gathered}
$$

В терминах функций $u(x, \lambda), v(x, \lambda)$ и их производных задачу можно достаточно легко свести к МПОСЗ для оператора вида (1), заданного в пространстве $E^{2}$.

Теорема 4. Пусть для любых попарно различных собственных значений $\lambda_{k}, \lambda_{j}, \lambda_{i}$ оператора (5) выполнены условия

$$
f_{1}\left(\lambda_{k}, \lambda_{j}, \lambda_{i}\right) \neq 0 \quad u \quad f_{2}\left(\lambda_{k}, \lambda_{j}, \lambda_{i}\right) \neq 0
$$

Тогда МПОСЗ имеет ровно шесть изолированных решений. 
Таким образом, четыре неизвестных параметра граничных условий можно восстановить с помощью четырех собственных значений оператора. Заметим, что если коэффициенты дифференциального выражения (5) - постоянные величины, то $f_{1}\left(\lambda_{k}, \lambda_{j}, \lambda_{i}\right)=$ $f_{2}\left(\lambda_{k}, \lambda_{j}, \lambda_{i}\right)$. При этом решения соответствующей МПОСЗ можно получить в явном виде.

В заключение отметим, что доказательства всех утверждений, сформулированных в данной статье, построены на теории вращений аналитических векторных полей (см. [4]) и методе продолжения по параметру.

\section{СПИСОК ЦИТИРОВАННОЙ ЛИТЕРАТУРЫ}

[1] И. А. Биргер, Техническая диагностика, Надежность и качество, Машиностроение, М., 1978. [2] F. V. Atkinson, Multiparameter Eigenvalue Problems. V. 1. Matrices and Compact Operators, Math. Sci. Engrg., 82, Acad. Press, New York, 1972. [3] Г. А. Исаев, Докл. АН СССР, 229:2 (1976), 284-287. [4] М. А. Красносельский, Г. М. Вайникко, П. П. Забрейко, Я.Б. Рутицкий, В.Я. Стеценко, Приближенное решение операторных уравнений, Наука, М., 1969.

Н. Ф. Валеев

Поступило

Башкирский государственный университет, г. Уфа

E-mail: valeevnf@mail.ru 